\title{
ANISOTROPY OF THE MICROWAVE BACKGROUND AND BIASED GALAXY FORMATION
}

\author{
Nick Kaiser \\ Institute of Astronomy \\ Madingley Road \\ Cambridge CB3 0HA \\ England
}

\begin{abstract}
Fluctuations in the microwave background will have been imprinted at $z \simeq 1000$, when the photons and the plasma decoupled. On angular scales greater than a few degrees these fluctuations provide a clear view of any primordial density perturbations, and therefore a clean test of theories which invoke such fluctuations from which to form the structure we see in the universe. On smaller angular scales the predictions are less certain: reionization of the gas may modify the spectrum of the primordial fluctuations, and secondary fluctuations may be generated.

Here I shall review some recent theoretical developments. A brief survey is made of the currently popular theories for the primordial perturbations, with emphasis on the predictions for large scale anisotropy. One major uncetainty in the predictions arises from the normalisation of the fluctuations to e.g. galaxy clustering, and much attention is given to the question of 'biased' galaxy formation. The effect of reionization on the primordial fluctuations is discussed, as is the anisotropy generated from scattering off hot gas in clusters, groups and galaxies.
\end{abstract}

\section{LARGE ANGLE ANISOTROPY}

There are various theoretical models for the primordial density fluctuations from which galaxies and other structure in the universe are often assumed to have grown. Perhaps the most popular assumption is that at very early times, possibly during an inflationary phase, there were laid down Gaussian, isentropic density fluctuations with the "Harrison-Zel'dovich" spectrum. Combining this with some assumption about the present material content of the universe one can calculate, in the manner first demonstrated by Peebles and Yu (1970), the residual fluctuations in the microwave background. These fluctuations arise from a combination of gravitational redshifts, Doppler redshifts and spatial variations of the radiation temperature at the epoch of last scattering (Bond and Efstathiou, 1984; Vittorio and Silk, 1984; see also the review article; Kaiser and Silk, 1986, for more complete references). For large angles, the fluctuations are dominated by a term which looks like (one third of) the Newtonian gravitational potential $\phi$ at the point of emission $\Delta T / T=-1 / 3 \phi\left(r_{\mathrm{em}}\right)$. (Sachs and Wolfe, 1967). In this approximation the microwave sky is simply a spherical secion of Gaussian 'flicker noise' since with the assumed spectrum $\left\langle\left|\phi_{k}\right|^{2}\right\rangle \propto k^{-3}$, and logarithmic intervals of wavenumber contribute equally to the total variance. If we make a set of measurements $\Delta_{i}$, each of which is a linear convolution of the sky temperature with some angular beam pattern, the probability distribution is

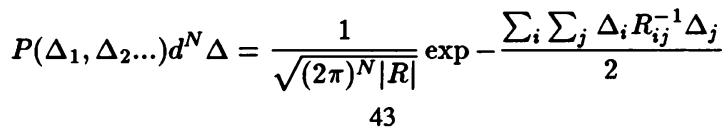


where

$$
R_{i j}=\left\langle\Delta_{i} \Delta_{j}\right\rangle=\frac{1}{9} \int d^{3} k W_{i}(\mathbf{k}) W_{j}(\mathbf{k})\left\langle\left|\phi_{k}\right|^{2}\right\rangle .
$$

Here $W_{i}(\mathbf{k})$ is the Fourier transform of the 3-dimensional window function $W_{i}(\mathbf{r})$ for the $i$ th observation, which is just the direct product of the angular beam pattern with the radial profile of the last scattering shell, and $\phi_{k}=4 \pi G \delta \rho_{k}$. These simple equations provide a complete statistical description of the fluctuations. The fluctuations are rich in harmonic content, and this probability distribution provides the best way to confront the theory with observation.

The 'flicker-noise' nature of the fluctuations is a property shared by many theoretical models. For instance, instead of isentropic fluctuations, in which the fluctuations in the number density of all particles are identical, one might opt for isocurvature fluctuations, in which at some initial time the perturbations in one component is cancelled by an opposite fluctuation in another component. The Sachs Wolfe term is now augmented by another contribution arising from the initial fluctuations in the specific entropy (Efstathiou and Bond, 1986; Suto, Sato and Kodama, 1985) so the amplitude will change, but otherwise equations 1 still apply.

Another alternative is to drop the assumption of Gaussian initial fluctuations. With cosmic string, step-like discontinuities are generated at points on the sky where the line of sight intercepts a transversely moving string (Gott, 1985; Kaiser and Stebbins 1984) The fluctuations in this case would not be described by the distribution function given above, but the amplitude of the fluctuations would still be approximately scale invariant.

For each of these choices for the initial fluctuations, further distinct models for galaxy formation can be constructed by choice of dominant component; e.g. massive neutrinos, cold dark matter of one form or another etc.. This will have implications for the spectrum of density perturbations only on scales below the maximum Jeans scale, but the amplitude at large angles will be affected indirectly through the normalisation procedure. What is needed for this is some measure of the mass fluctuations, ideally in the regime where linear theory can still be applied to extrapolate back to the initial amplitude. We turn now to this question of normalisation which is probably the weakest link in the whole calculation.

\section{NORMALISATION AND BIASED GALAXY FORMATION}

A commonly used datum for normalising the initial perturbation amplitude is the variance of galaxy counts in spheres of radius $8 \mathrm{Mpc} / \mathrm{h}$, which is roughly unity. The motivation for this choice is that the fluctuations on these scales are well determined observationally, but should still be adequately described by linear theory. If one believed that the fluctuations in galaxy number were representative of the mass fluctuations, then the normalisation would be straightforward. However, if the universe has closure density, as all the models discussed here presuppose, then it must be that in the dense regions such as clusters which are chosen for virial analysis, galaxies are overabundant by a factor $(M / L)_{\text {universe }} /(M / L)_{\text {cluster }} \simeq 3-5$. In 'biased' theories of galaxy formation it is proposed that galaxy formation is enhanced in regions which were initially overdense, and it then follows that the fluctuations in galaxy counts on large scales would exceed the true density fluctuations by some factor $b$.

One rather ad hoc model for biasing is to associate galaxies with the high initial density peaks (see Bardeen et al., 1986). With this model one finds that in order to get a non-linear bias of galaxy formation in clusters of the right amplitude results in a linear bias for long-wavelength modes of $b \simeq 2$. Perhaps the most implausible feature of this model is the sharp division of perturbations into galaxies and 'failed' galaxies according to the threshold value $\nu$. This threshold model may be applicable to cluster clustering, where we see a snapshot of the clustering hierarchy, and it is reasonable to assume that a region destined to collapse just later than the present would not get into a cluster catalogue, but for galaxies one has the feeling that the lower amplitude perturbations are just 'waiting in the wings' and would make luminous galaxies, but just a little later. A sharp 
cut-off might arise if there is some rapidly imposed negative feedback which terminates galaxy formation once a small fraction of the potential galaxies have formed, but no obvious candidate for the regulating influence has emerged.

Here we explore a class of models in which the luminosity of a galaxy is a smoothly varying function of the physical parameters of the dark halo, and the sharp threshold (if any) is imposed by the observer who constructs a magnitude limited catalogue.

\section{(More or Less) Natural Biasing}

Consider a Gaussian hierarchical model characterised by an initial rms density fluctuation as a function of mass scale $\sigma(M)$. One can imagine integrating forward from a realisation of this spectrum into the non-linear regime, and applying a cluster or group finding algorithm, to obtain, at any epoch $y \equiv 1+z$, a cumulative mass spectrum of dark matter concentrations (which we will refer to as 'halos') $N(>M, y)$. We will assume that the form of this function is like that given by the Press and Schechter (1974), but we will not need very detailed knowledge. It will also be assumed, following the spherical collapse model, that shells of matter which have recently virialised have a density contrast of around 200, and that the group finding algorithm has been adjusted to select clumps with this density contrast. A convenient quantity in what follows is the comoving number density of halos per logarithmic mass interval: $N_{M}(M, y) \equiv \partial N(>M, y) / \partial \ln M$. At this crude level of description the halos form a two parameter family.

We now postulate a simple model for galaxy formation in which the stellar luminosity which is generated in a halo has a simple power law dependence on the collapse time and on the mass collapsed:

$$
L(M, y)=M^{\alpha} y^{\beta} .
$$

This is a fairly flexible model since any thermodynamic variable characterising the halo (e.g. temperature, pressure etc.) can be written as a product of powers of $M$ and $y$. The model is still ad hoc in that with our present understanding of galaxy formation it is not feasible to derive the parameters $\alpha$ and $\beta$. What is attempted here is a simple characterisation of the dependence of luminosity on halo properties which might plausibly reflect the actual physics governing star formation, and one can at least hope to be able to constrain the parameters $\alpha$ and $\beta$.

We shall now estimate the biasing of these galaxies. That is, we calculate the response of this system to long-wavelength perturbations. We will consider two applications: For sufficiently small amplitude imposed perturbations $\Delta_{\mathrm{B}}=\Delta \rho / \rho$ we obtain a linear response for the perturbation in the (Eulerian) density of objects of a chosen range of luminosity $(\Delta N / N)_{L}=b \Delta_{\mathrm{B}}$. It is this factor which is used to correct the observed variance of galaxy counts to the fluctuations in the underlying matter distribution. Another quantity of interest is the amount by which the abundance of galaxies per unit mass would differ from the mean value inside a cluster which grew from a density perturbation $\Delta_{C L}$. We will denote this by $B$; any viable biasing scheme must give $B \simeq 3-5$ in order to solve the ' $\Omega$-problem'.

Consider then a realisation of the assumed initial random field, make a replica and add some long-wavelength density perturbation $\Delta_{B}$ to make a perturbed realisation. The primary effect of $\Delta_{B}$ is simply to enhance or retard the collapse times of the objects, thereby modifying the luminosity of each object:

$$
L \rightarrow L^{\prime}=L\left(1+\Delta_{\mathrm{B}} / \Delta_{\mathrm{OBJ}}\right)^{\beta},
$$

where $\Delta_{\mathrm{OBJ}}$ is the initial density perturbation for the object. We see immediately that a positive value of $\beta$ is required to give a positive bias.

There is a minor problem with this analysis that for any chosen luminosity there will be a range of collapse times, and hence a range of values for $\Delta_{\mathrm{OBJ}}$. However, for the values of $\alpha$ and $\beta$ of interest here, this range is limited, and consequently the objects form, to a first approximation, a 1-parameter family. What happens in practice is that $N_{\mathrm{L}} \equiv \partial N(>L, y) / \partial \ln L$, the comoving 
number density of objects per log-interval of $L$, rises very steeply at first, reaches a maximum and then decreases. For any $L$ there is a well defined epoch $y_{\max }(L)$ when $N_{\mathrm{L}}$ peaks, and if most of these objects survive as distinct galaxies, $N\left(>L, y_{\max }(L)\right)$ should provide a useful 1-parameter distribution which can be compared with observations.

An indication of the strength of biasing expected with the cold dark matter spectrum is given if we consider scale free initial conditions $\sigma \propto M^{-(n+3) / 6}$. With our model for $L(M, y)$ we have $L \propto M_{*}^{\alpha} y^{\beta} \propto y^{(\beta-6 \alpha /(n+3))}$ and $N_{\mathrm{L}} \propto M_{*}^{-1}$ and hence

$$
N_{\mathrm{L}}=\left(L / L_{0}\right)^{-1 /(\alpha-\beta(n+3) / 6)}
$$

and in a perturbed region

$$
N_{\mathrm{L}}^{\prime}=\left(L / L_{0}^{\prime}\right)^{-1 /(\alpha-\beta(n+3) / 6)}
$$

with $L_{0}^{\prime}=L_{0}\left(1+\Delta_{\mathrm{B}} / \Delta_{\mathrm{OBJ}}\right)^{\beta}$ and so

$$
\left(\frac{N^{\prime}}{N}\right)_{L}=\left(1+\Delta_{\mathrm{B}} / \Delta_{\mathrm{OBJ}}\right)^{\frac{\beta}{(\alpha-(n+3) \beta / 6)}}
$$

with linearised version

$$
\Delta N / N=\frac{\beta}{(\alpha-(n+3) \beta / 6)} \frac{\Delta_{\mathrm{B}}}{\Delta_{\mathrm{OBJ}}} .
$$

In these equations the number densities refer to Lagrangian coordinates. To get the Eulerian number density one must multiply by the (Eulerian) matter density.

Consequences of equations 4 are that with $\alpha$ and $\beta$ fixed the bias will increase with increasing $L$ (as $L$ increases $\Delta_{\mathrm{OBJ}}$ decreases and the spectral index $n$ also increases). Note however that the bias in this model is positive for all objects. This is quite unlike the result obtained if we just take a 'snapshot' of the halos at a given epoch. In that case one finds that one obtains a positive bias for the most massive objects at the expense of a negative bias for the smaller objects.

Equations 4 allow one to calculate the bias for any class of object. One particularly interesting class are those objects for which the net-luminosity density per $\log$ interval of $L$ is maximised. Crudely, one can say that these objects dominate the light. These have $N_{\mathrm{L}} \sim L^{-1}$, and one obtains for the of these objects $N^{\prime} / N=\left(1+\Delta_{\mathrm{B}} / \Delta_{\mathrm{OBJ}}\right)^{\beta}$, which is also the net luminosity density bias.

An interesting special case of the model (equation 2) is to assume that the luminosity varies as the 4th power of the halo rotation velocity. Since $v^{2} \propto M^{2 / 3} y$ this is equivalent to $\alpha=4 / 3, \beta=2$. This choice has been dubbed 'natural biasing' and has been explored via numerical simulations (Frenk et al., 1987). From the expression given above for $N^{\prime} / N$, it would appear that in order to get a reasonable $B$, one would need to have $\Delta_{\mathrm{CL}} / \Delta_{\mathrm{OBJ}}$ approaching unity, or equivalently that on average these galaxies only form just before clusters. While a late formation epoch seems to be indicated from the numerical simulations, a different picture comes from the statistics of the initial perturbations. For example, going from a cluster mass to a mass 1000 times smaller the rms fluctuations increase by about a factor 6 . One would therefore expect that if $\nu \sigma\left(M_{\mathrm{CL}}\right)$ initial perturbations on a cluster scale make clusters, then the same $\nu$ perturbations on the smaller mass scale would have made objects at $1+z \simeq 6$. These objects would have a comoving number density 1000 times that of clusters and velocity dispersions smaller by a factor $\sqrt{6} / 10$, but these are roughly the characteristics of ordinary bright galaxies. With $\Delta_{\mathrm{CL}} / \Delta_{\mathrm{OBJ}} \simeq 1 / 6$, the bias would be much too weak. Inspection of the Press-Schechter mass function for this spectrum reveals that while objects with galaxy-like velocity dispersions should have formed at $1+z \simeq 6$ with about the right abundance, the number of objects with that velocity dispersion remains roughly constant to considerably later times, and these later collapsing objects would be more strongly biased. Another ameliorating factor is that the brightest galaxies have a stronger bias, so for a magnitude limited catalogue one would predict a bigger effect.

While the indications are that the $\beta=2$ model gives too weak a bias, it is easy to get an adequate strength by postulating a larger value for $\beta$. We shall now ask what constraints on $\alpha$ and $\beta$ arise from the observed properties of galaxies. 
If we assume that the final velocity dispersion of the stars in these galaxies reflects that of the parent halo then we can constrain the model. The requirement that the objects produce a luminosityvelocity dispersion relation $L \propto v^{\gamma}$ gives the constraint

$$
\alpha-(n+3) \beta / 6=\gamma(1-n) / 12
$$

If $\alpha$ satisfies equation 5 , and $\beta=\gamma / 2$, then all of the objects lie exactly on the $L-v$ relation. If $\beta \neq \gamma / 2$ then the mean luminosity velocity dispersion relation comes out right but there will be scatter about this line, which one could use to constrain $\beta$. One interesting value for $\beta$ is zero, corresponding to an unbiased 'what you see is what you get' model in which mass turns into light with equal efficiency in all halos; arguably the most natural choice for $\beta$. Such a value for $\beta$ would give very large scatter about the mean $L-v$ relation: Consider 1-sigma and 2-sigma perturbations on the same mass scale. These will collapse at different times, and will have $v^{4}$ differing by a factor 4 , but by assumption would have the same luminosity. This is much larger than the observed scatter in the Tully-Fisher or Faber-Jackson relations, so this unbiased model would seem to be excluded. For large values of $\beta$, it turns out that the scatter is not so large and such models are more difficult to rule out.

A more hopeful prospect for testing high- $\beta$ models comes from the systematic variation of the $L-v$ relation with environment that is predicted. One finds an offset in the mean line

$$
(\Delta L / L)_{v}=(\gamma / 2-\beta)\left(\Delta_{\mathrm{B}} / \Delta_{\mathrm{OBJ}}\right)
$$

thus one should find, for galaxies of a given velocity dispersion, an inverse correlation of luminosity with local density.

To summarise: We have found the perhaps not surprising result that, in a model in which the luminosity generated in a halo of a given mass is an increasing function of collapse redshift, one obtains a positive bias (more galaxies per unit mass in overdense regions). The model contains a tunable parameter $\beta$ which controls the strength of the bias. For the galaxies to satisfy the luminosity-velocity dispersion relation requires a non-trivial dependence of luminosity on halo mass. A special case of the model is the 'natural' biasing model $(\alpha=4 / 3, \beta=-2$ in our notation), but the bias turns out to be rather weak. Models with larger $\beta$ give a large bias and result in the testable rediction that the zero point of the $\dot{L}-v$ relation should vary systematically with local density as indicated by equation 6 .

The statistics of the models proposed here are similar to the sharp threshold 'high- $\nu$ ' model. For large $\beta$ they become indistinguishable, with $\beta$ playing the role of $\nu^{2}$, and one has therefore a similar relation between $B$, the enhancement in the specific luminosity density of virialised systems, and $b$, the amplification factor for long-wavelength modes, suggesting that one should normalise to rms mass fluctuations in $8 \mathrm{Mpc} / \mathrm{h}$ spheres of about $50 \%$.

\section{Alternative Normalisation}

It is possible to normalise the initial amplitude more directly, at least in principle, using the observed properties of rich clusters where one see the clustering of the mass directly from virial analysis and $\mathrm{X}$-ray studies. If a region of comoving radius $R$ turns round and virialises at a density contrast $\sim 200$, the final radius is $R_{f} \simeq 0.17 R$ and the circular velocity would be $v_{c}=10 H R_{f}$. These properties provide a reasonable match to those of real rich clusters (richness class $>1$ say) for $R \simeq$ $8 \mathrm{Mpc} / \mathrm{h}$. These clusters contain $4 \pi R^{3} / 3 n \simeq 1 \%$ of the mass, and so should have formed from $\nu \simeq 2.5$ fluctuations. Invoking the spherical collapse model to find the initial overdensity required to form a virialised entity by the present, one obtains for the rms mass fluctuation in $8 \mathrm{Mpc} / \mathrm{h}$ spheres 
$\sigma_{8} \simeq 1.7 / 2.5 \simeq 70 \%$, corresponding to $b \simeq 1.4$; somewhat smaller than that suggested from the considerations of biased galaxy formation.

\section{REIONISATION AND SECONDARY FLUCTUATIONS}

Most calculations of the anisotropy arising from primordial fluctuations assume that the universe remains neutral after decoupling. If the universe is reionized at sufficiently high redshift that the optical depth exceeds unity then the last scattering shell moves to lower redshift. This has important consequences for the anisotropy on scales less than a few degrees. If reionisation occurs later the primordial perturbations will be preserved, but will be augmented by additional anisotropies arising from the scattering of photons of hot ionised gas in cluster, group and galaxy potential wells.

\section{Reionisation and Secondary Anisotropy}

If the gas is reionized at redshift $z \sim 50$ or greater, the optical depth back to decoupling is large, and primordial fluctuations are efficiently erased. Secondary fluctuations will be generated in the new last scattering shell from the motion of the gas associated with the growing density perturbations. Analysing this to 1st order in perturbation theory, one finds that there is almost complete cancellation of the Doppler effect for perturbations of wavelength $\lambda \ll c t_{1 s}$, the horizon size at last scattering (Kaiser, 1984). The dominant contribution is of order $\Delta T / T \sim\left(\lambda / c t_{\mathrm{ls}}\right)^{5 / 2} \Delta \rho / \rho$.

More recently Vishniac (1987) has shown performed an analysis to 2nd order in the perturbation. He finds that if there are perturbations on a smaller scale, these break the cancellation. If we consider a simple model with density fluctuations on two scales $\lambda_{1}, \lambda_{2}$ with $\lambda_{1}>\lambda_{2}$, one finds

$$
\frac{\Delta T}{T} \sim \frac{(\Delta \rho / \rho)_{2}}{\sqrt{N_{2}}} v_{1},
$$

where $N_{2}$ is the number of the smaller wavelengths through the last scattering shell. The effect is analysed in detail in Vishniac's paper, but it is useful to note that, for the cold dark matter spectrum, the first term peaks on a galactic scale, where the effective spectral index is $n=-2$, and the second term peaks on roughly a cluster scale where $n \simeq-1$. This suggests that compared with the standard calculation, in a reionized universe the fluctuations would be substantially decreased on scales below about a degree or so, but would appear grainy on angles corresponding to a galaxy scale $\sim$ an arc minute.

\section{Secondary Fluctuations from the S-Z Effect}

Fluctuations in the microwave background will arise from scattering of photons off the hot gas in clusters of galaxies (Sunyaev and Zeldovich 1970), and much effort has been devoted to the detection of this effect in individual clusters (Birkinshaw and Gull 1984). Ostriker and Vishniac (1986) have drawn attention to the possibility that at the epoch of galaxy formation, the hot primordial gas confined in the galactic halos will also produce a general background of fluctuations, but on smaller angular scales. The recent claims of detection of anisotropy on these scales with the VLA at levels similar to the predictions (Fomalont et al., 1986; Martin and Partridge, 1986) have therefore created much interest. Here I will discuss the predictions for the amplitude, angular scale dependence and statistical distribution of the SZ fluctuations for the cold dark matter model.

In this model it is found that at any epoch one finds virialized structures with a wide range of masses, with a progression to higher masses with increasing time. In the Raleigh-Jeans part of the spectrum the SZ deficit is the integral of the electron pressure along the line of sight

$$
\frac{\Delta T}{T}=-2 y=-2 \int d l n_{\mathrm{e}} \sigma_{\mathrm{T}}\left(k T_{\mathrm{e}} / m_{\mathrm{e}} c^{2}\right)
$$


Unfortunately numerical methods are at present unable to model both the gas and the dark matter evolution, so in order to predict the statistical properties of the electron pressure one must resort to some approximation for the gas dynamics. Here we assume that at any epoch the virialized condensations form a one parameter (mass or virial temperature) family, and that the distribution function $n(M, z)$ (the comoving number density of objects existing at redshift $z$ and with masses in the range $M ; M+d M$ being $n(M, z) d M)$ may be calculated from the formula given by Press and Schechter (1974). We further assume that the gas is shock heated to the virial temperature on infall and then sits in hydrostatic equilibrium in the dark-matter potential well. The detailed assumptions adopted and the calculational procedure are described in Cole and Kaiser (1987), here we make some simple estimates to identify the range of masses and redshifts that will dominate the effect and draw some general conclusions.

The SZ decrement from a single object of physical radius $R$ is $\Delta T / T \sim n_{\mathrm{e}} R T_{\mathrm{e}} \propto M(1+z)^{3}$, since $T_{\mathrm{e}} \sim M / R$ and for systems which have just virialized the density is just some multiple of the background density. This object will subtend an angle $\theta(M, z) \propto M^{1 / 3} g(z)$, where $g(z) \propto z^{-1}$ at low $z$ and $g$ becomes asymptotically constant at high $z$. The total variance in the microwave background fluctuations can be written as a double integral over masses and redshifts. Consider first the fluctuations induced by objects in some fixed small redshift interval; each of these removes a net flux $\Delta F \sim \theta^{2} \Delta T / T \propto M^{5 / 3}$, so the total sky variance, assuming a Poissonian distribution of clusters, is

$$
\left(\frac{\Delta T}{T}\right)^{2} \sim \int d M n(M)(\Delta F)^{2} \sim \int d M n(M) M^{10 / 3}
$$

A general feature of hierarchical models with Gaussian initial density fluctuations is that the slope of the mass function increases towards high masses, reflecting the exponentially falling tail of the Gaussian distribution. We infer from this that the logarithmic mass range which produces the greatest fluctuations will be at a relatively high mass $M_{\max }$, where $n(M)$ is falling very rapidly $n\left(M_{\max }\right) \sim M^{-13 / 3}$. At any epoch then, we expect that the fluctuations will be dominated by very rare, massive condensations, and this has important consequences for the statistics of the fluctuations: were the more typical " 1 -sigma" condensations dominant one would have many independent condensations along any line of sight and the central limit theorem would indicate a roughly Gaussian sky pattern. With the dominance by rare objects, one expects a more spiky, non-Gaussian pattern.

It is unrealistic to assume that the objects have a strictly Poissonian distribution. However, for our purposes it is a reasonable approximation. Even though high- $\nu$ objects will have a large correlation function $\xi$, what is important for the statistics of the microwave fluctuations is whether one gets correlated objects in the same or neighbouring beams. This is determined by the product of $\xi$ and the number density $n$, and this becomes exponentially small for high- $\nu$.

Consider now the redshift dependence. A guide to what epochs are most important can be obtained from consideration of models with a power-law initial power spectrum $P(k) \propto k^{n}$. Defining $f_{M}$ to be the fraction of mass per $\log$ interval of mass: $f_{M}(M, z) \equiv M^{2} n(M, z)$, the scale-independence of the intial fluctuations converts to a temporal self-similarity, $f_{M}(M, z)=F\left(M / M_{*}(z)\right)$ where $M_{*}(z) \propto(1+z)^{-6 /(n+3)}$. In such a model, the dominant mass identified above will scale with redshift just like $M_{*}$, and the decrement from any one of these objects scales as $(\Delta T / T)_{*} \propto(1+z)^{(3 n+3) /(n+3)}$. The total variance due to those objects in a logarithmic interval of redshift is the square of $(\Delta T / T)_{*}$ multiplied by $f_{\text {sky }}$, the fraction of sky covered by these objects. For $z \gg 1$ this scales as $f_{\text {sky }} \propto$ $(1+z)^{(1-n) /(2 n+6)}$, and combining these gives a total variance

$$
f_{\text {sky }}\left(\frac{\Delta T}{T}\right)^{2} \propto(1+z)^{(11 n+13) /(2 n+6)} .
$$

We see from this that with $n>-13 / 11$, the fluctuations diverge towards high $z$, whereas with $n<-13 / 11$ the redshifts of order unity will dominate. For the cold dark matter spectrum, the spectral index varies slowly from $n=-3$ at small masses to $n=1$ at the high mass end. The effective spectral index has the critical value $-13 / 11$ around the mass scale of clusters, and we find 
that with the Press-Schechter approximation for the mass spectrum, the critical slope for $n(M)$ is obtained for those objects which contain about $1 \%$ of the mass, which again is similar to the mass fraction contained in Abell clusters. We conclude then that the objects which dominate the SZ effect in this model are objects with masses similar to rich clusters and at fairly recent epochs. The constribution from galaxy mass perturbations in particular is very small.

Shown below is a simulation of a patch of the microwave sky $16^{\prime}$ square, obtained by convolving the Press-Schechter distribution with 'template' clusters with profiles like isothermal spheres.

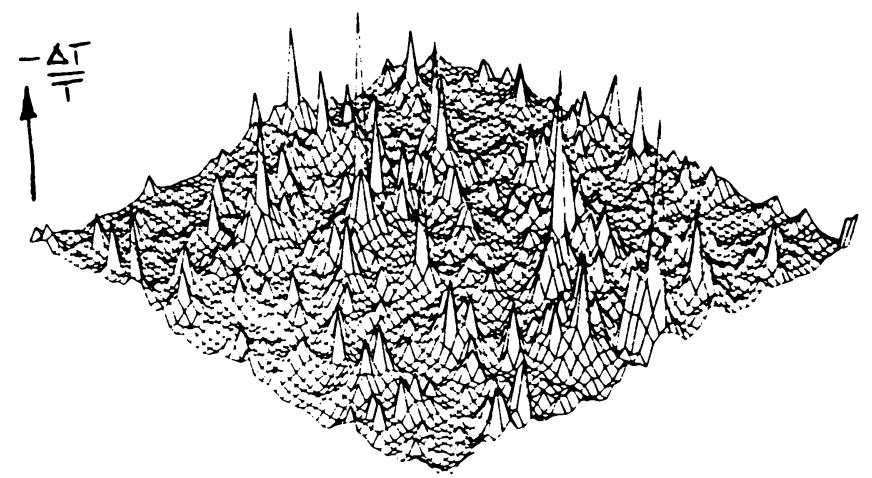

The total $\mathrm{rms} \Delta T / T \simeq 10^{-5}$, but this depends quite sensitively on the normalization. Consider the contribution from clusters with a known number density. Even if the gas fraction and its distribution relative to the dark matter were perfectly known, the net flux decrement varies as the fifth power of the cluster velocity dispersion $\left(M \propto \sigma^{3}\right.$ for objects of the same density). This means that the final amplitude of the fluctuations must inevitably be quite uncertain. Interesting features which are insensitive to the normalisation are the slow dependence of the sky variance on angular scale (noted also by Dick Bond), and the strongly non-Gaussian character.

\section{REFERENCES}

Bardeen, J.M., Bond, J.R., Kaiser, N. and Szalay, A., 1986. Ap.J., 304, 15.

Birkinshaw, M. and Gull, S.F. 1984. Nature, 309, 34.

Bond, J.R. and Efstathiou, G.P., 1984. Ap.J., 285, L45.

Cole, S. and Kaiser, N., 1987. In preparation.

Efstathiou, G.P. and Bond, J.R., 1986. M.N.R.A.S., 218, 103.

Frenk, C.S., White, S.D.M., Davis, M. and Efstathiou, G.P., 1987. Preprint.

Fomalont,E.B.,Kellermann,K.I.,Weistrop,D.,and Wall,J.V. 1986. Highlights of Astronomy, 7, 367.

Gott, J.R., 1985. Ap.J., 288, 422.

Kaiser, N. 1984. Ap.J., 282, 374.

Kaiser, N. and Silk, J., 1986. Nature, 324, 529.

Kaiser, N. and Stebbins, A., 1984. Nature, 310, 391.

Martin, B. and Partridge, B., 1986. Preprint.

Ostriker, J.P.and Vishniac, E.T., 1986. Ap.J., 306, L51.

Peebles, P.J.E. and Yu, J.T., 1970. Ap.J., 162, 816.

Press, W.H. and Schechter, P., 1974. Ap.J., 187, 425.

Sachs, R.K. and Wolfe, A.M., 1967. Ap.J., 147, 73.

Sunyaev, R.A. and Zel'dovich, Ya.B. 1970. Astrophys. and Space Sci., 7, 3.

Suto, Y., Sato, K. and Kodama, H., 1985. Ap.J., 292, L1.

Vittorio, N. and Silk, J., 1984. Ap.J., 285, L35.

Vishniac, E.T., 1987. Preprint. 